\title{
Isolation of a novel histone deacetylase reveals that class I and class II deacetylases promote SMRT-mediated repression
}

\author{
Hung-Ying Kao, ${ }^{1}$ Michael Downes, ${ }^{1}$ Peter Ordentlich, ${ }^{1}$ and Ronald M. Evans ${ }^{2}$ \\ Howard Hughes Medical Institute, Gene Expression Laboratory, The Salk Institute for Biological Studies, \\ La Jolla, California 92037 USA
}

\begin{abstract}
The transcriptional corepressor SMRT functions by mediating the repressive effect of transcription factors involved in diverse signaling pathways. The mechanism by which SMRT represses basal transcription has been proposed to involve the indirect recruitment of histone deacetylase HDAC1 via the adaptor mSin $3 \mathrm{~A}$. In contrast to this model, a two-hybrid screen on SMRT-interacting proteins resulted in the isolation of the recently described HDAC5 and a new family member termed HDAC7. Molecular and biochemical results indicate that this interaction is direct and in vivo evidence colocalizes SMRT, mHDAC5, and mHDAC7 to a distinct nuclear compartment. Surprisingly, HDAC7 can interact with $\mathrm{mSin} 3 \mathrm{~A}$ in yeast and in mammalian cells, suggesting association of multiple repression complexes. Taken together, our results provide the first evidence that SMRT-mediated repression is promoted by class I and class II histone deacetylases and that SMRT can recruit class II histone deacetylases in a $\mathrm{mSin} 3 \mathrm{~A}$-independent fashion.
\end{abstract}

[Key Words: Nuclear receptor; corepressor; SMRT/N-CoR; histone deacetylase; mHDAC7; transcriptional repression]

Received October 21, 1999; revised version accepted November 29, 1999.

Nuclear hormone receptors are sequence-specific and ligand-dependent transcription factors that control cell proliferation, differentiation, and animal physiology (Mangelsdorf and Evans 1995; Mangelsdorf et al. 1995). They are structurally related and contain two evolutionarily conserved modules, the DNA-binding (DBD) and ligand-binding domains (LBD). Several receptors including retinoic acid and thyroid hormone receptors function as potent repressors in the absence of ligands and as activators upon ligand binding. Intensive studies on the mechanism of transcriptional activation by nuclear hormone receptors led to the identification of coactivators including CBP/p300 and PCAF, as well as the p160 family proteins (SRC-1; GRIP1/TIF2; ACTR/RAC3/P/CIP) (Onate et al. 1995; Hong et al. 1996; Kamei et al. 1996; Yao et al. 1996; Chen et al. 1997; Torchia et al. 1997; Blanco et al. 1998). Among these, CBP, PCAF, and SRC$1 /$ ACTR have been shown recently to possess intrinsic histone acetyltransferase activity, consistent with a role for histone acetylation in transcriptional activation (Bannister and Kouzarides 1996; Ogryzko et al. 1996; X.J. Yang et al. 1996; Chen et al. 1997; Spencer et al. 1997). Several corepressors for nuclear receptors including

\footnotetext{
${ }^{1}$ These authors contributed equally to this work.

${ }^{2}$ Corresponding author.

E-MAIL evans@salk.edu; FAX (858) 455-1349.
}

SMRT, N-CoR, SUN-CoR, and Alien have also been identified (Chen and Evans 1995; Horlein et al. 1995; Zamir et al. 1996; Dressel et al. 1999; Ordentlich et al. 1999). SMRT and N-CoR were identified by yeast twohybrid screens with nuclear receptors. Both proteins are large and possess at least four autonomous repression domains. In addition to nuclear receptors, functional associations between SMRT/N-CoR with other transcription factors including CBF1/RBPJK, PLZF, BCL6, MyoD, Bach2, and Pbx1 have been demonstrated (Dhordain et al. 1997; Hong et al. 1997; He et al. 1998; Huynh and Bardwell 1998; Kao et al. 1998; Lin et al. 1998; Muto et al. 1998; Wong and Privalsky 1998; Asahara et al. 1999; Bailey et al. 1999), suggesting that corepressors, like coactivators, may function as signaling integrators to control cell fate. Several lines of evidence suggest that the mechanism underlying the repressive activity of SMRT and N-CoR corepressors is manifested through their recruitment of a histone deacetylase complex containing mSin3A and HDAC1 (Alland et al. 1997; Hassig et al. 1997; Heinzel et al. 1997; Laherty et al. 1997; Nagy et al. 1997; Zhang et al. 1997). Recruitment of acetylase/ deacetylase complexes by coactivators/corepressors is thought to cause a local change in the chromatin structure, resulting in either activation or repression of gene transcription.

In yeast Sacchromyces cerevisiae, two distinct histone deacetylase complexes have been characterized /Carmen 
et al. 1996; Rundlett et al. 1996). Histone deacetylase-B (HDB) is a $600-\mathrm{kD}$ complex, which contains the $\mathrm{Rpd} 3$ protein. Histone deacetylase-A (HDA) is a $350-\mathrm{kD}$ complex and contains yeast $\mathrm{Hda} 1$ and the related Hos1, Hos2, and Hos3. Homology studies indicate that the Hda1-related deacetylases are structurally distinct from Rpd3. Rpd3 appears to be most related to mammalian class I deacetylases HDAC1, HDAC2, and HDAC3. Class II mammalian histone deacetylases (HDAC4, HDAC5, and HDAC6) have been identified recently, which are structurally related to yeast Hdal (Fischle et al. 1999; Grozinger et al. 1999; Verdel and Khochbin 1999). These family members are large in size (from 1085 amino acids to 1216 amino acids) and are able to deacetylate histones in vitro. HDAC4 and HDAC5 (also known as mHDA1) are highly homologous $151 \% / 63 \%$ in identity/homology) and contain a conserved carboxy-terminal deacetylase domain (89\% amino acid identity). Intriguingly, HDAC6 (also assigned as mHDA2) has two catalytic domains at the amino-terminal, which have been suggested to form an intramolecular dimer. Whereas HDAC4 has been shown to coprecipitate with HDAC3 and RbAp48, HDAC5 appears to associate with at least HDAC3 (Grozinger et al. 1999). Furthermore, Northern blot analyses indicate that the tissue distribution patterns of family members are quite distinct. Numerous studies have indicated that the HDAC1/HDAC2 complexes are recruited to promoters by sequence-specific DNA-binding transcription factors (W.M. Yang et al. 1996; Emiliani et al. 1998; Doetzlhofer et al. 1999; Iavarone and Massague 1999; Radkov et al. 1999). A recent report suggests that HDAC4 associates with and represses the MEF2 transcription factor (Miska et al. 1999). However, the role of the HDAC4-HDAC6 family of histone deacetylases in transcription is largely unknown.

In this study, we provide evidence that the HDAC4, HDAC5, and HDAC7 deacetylases may mediate nuclear receptor repression. Significantly, we describe a novel family member, HDAC7, and characterize its interaction with SMRT/N-CoR and mSin3A, its biochemical properties, and subcellular localization. The findings described here indicate that both classes of histone deacetylases can collectively contribute to SMRT/NCoR action and that at least some deacetylases may associate directly with SMRT/N-CoR in a mSin3A-independent fashion.

\section{Results}

\section{SMRT interacts with class II deacetylases in yeast}

To determine whether SMRT repression may be mediated through interaction with factors other than mSin3A, a yeast two-hybrid screen was conducted with SMRT repression domains III and IV (designated previously as SRD I and SRD II) as a bait. This screen led to the isolation of several interacting clones whose products encode members of the newly described class II family of mammalian deacetylases. The majority of clones identified express the carboxyl terminal histone deacety- lase domain of the recently identified HDAC5 (Grozinger et al. 1999; Verdel and Khochbin 1999). This protein was also identified by others during the progress of this screen. Two of the clones, however, encode a novel class II histone deacetylase, which we termed HDAC7.

A yeast $\beta$-galactosidase liquid assay was carried out to evaluate the interaction between SMRT and mHDAC5 and mHDAC7. Clone 32A, which encodes the carboxyterminal deacetylase domain of HDAC5 was tested first. In the presence of the bait construct GAL DBD-SMRT (RD III + RD IV), increased reporter activity is observed, indicating an association between SMRT and mHDAC5 (Fig. 1A). Interestingly, deletion of the carboxy-terminal sequence to the HDAC5 deacetylase domain dramatically decreased reporter activity (Fig. 1B). Similar results were obtained when clone 23C, which encodes the carboxy-terminal sequence of mHDAC7, was used.

\section{A}
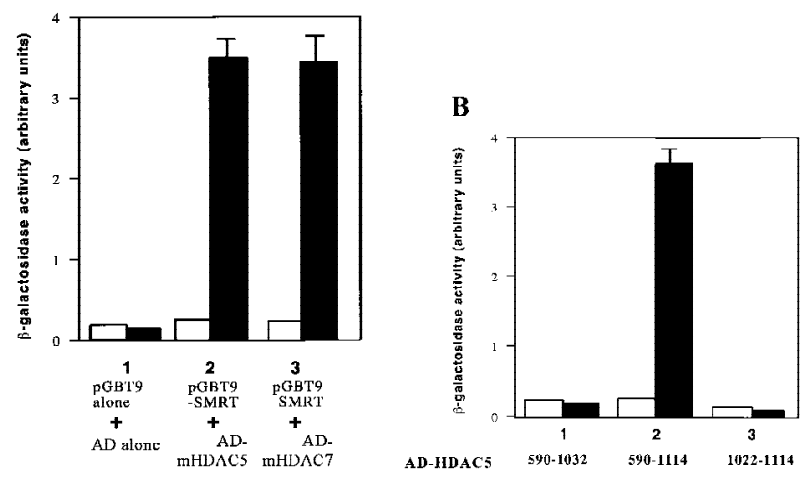

C

-Trp-Leu -Trp-Leu-His + $40 \mathrm{mM}$ 3-AT
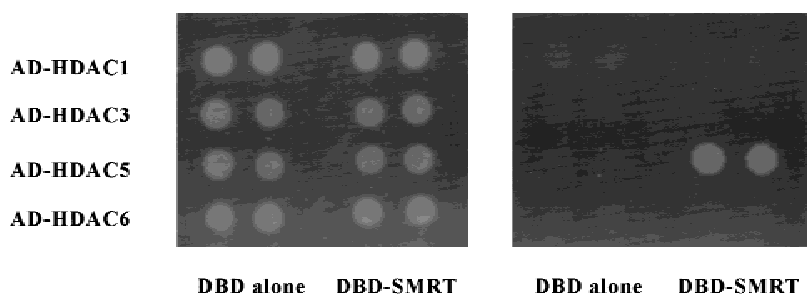

Figure 1. SMRT repression domains III and IV interact with deacetylase domain of the newly described deacetylases. (A) Quantitation of yeast two-hybrid assays between SMRT and HDAC5 and HDAC7. Yeast cells were cotransformed with the indicated plasmids. Two viable transformants were picked for liquid $\beta$-galactosidase activity. (Open bars) $\mathrm{AD}$; (solid bars) ADHDAC. The number represents average of duplicates from two colonies. (B) SMRT interaction requires amino acid carboxyterminal to the deacetylase domain of mHDAC5. Yeast method as described in $A$. (Open bars) pGBT9 alone; (solid bars) pGBT9SMRT. (C) SMRT RD III and RD IV do not interact with HDAC1 or mHDAC6. The bait construct pGBT9-SMRT (RD III + RD IV) was cotransformed with GAL AD fusion to various HDAC constructs into yeast strain Y190. After 3 days, two isolated colonies were picked, resuspended in water, and 103 cells were dropped on both -Trp-Leu and -Trp-Leu-His + $40 \mathrm{~mm}$ 3-AT plates. Pictures were taken after 3 days. 
Examination of these interacting clones indicated that the minimal SMRT interacting domain encompasses the beginning of the histone deacetylase domain. Because the catalytic cores of the histone deacetylases are similar to each other, we asked whether SMRT could also interact with other histone deacetylases such as HDAC1, HDAC3, and mHDAC6 [also designated mHDA2 (Verdel and Khochbin 1999)]. A series of AD-HDAC constructs were generated for interaction assays in yeast. Transformants harboring both plasmids were picked and patched onto nutrient selection plates. Only cells that contain plasmids carrying an interacting partner are able to activate reporter gene HIS3 and therefore are viable in the selection plate-Trp-Leu-His + $40 \mathrm{~mm}$ 3-aminotriazole (3-AT). As shown in Figure 1C, in contrast to mHDAC5 and mHDAC7, the deacetylase domains of HDAC1, HDAC3, and mHDAC6 do not interact with SMRT in a yeast two-hybrid assay. These results indicate that association with HDAC5 and HDAC7 is specific and not an intrinsic property of the deacetylase domain.

\section{Isolation of mHDAC7, a new member \\ of the novel histone deacetylases}

To obtain the full-length cDNAs of mHDAC5 and mHDAC7, a mouse brain library was probed with DNA fragments from the yeast library clones. Overlapping clones were obtained for both mouse mHDAC5 and $\mathrm{mH}$ DAC7. The full-length mHDAC5 contains an additional 123 amino acids in its amino-terminal compared with previously reported mHDA1. In addition, the previously reported mHDA1 encodes a 991 amino acid polypeptide that is 131 amino acids shorter than its human homolog HDAC5 and, therefore, is likely an incomplete cDNA or a spliced variant (Verdel and Khochbin 1999). Sequence comparison of human and mouse HDAC5 reveals an overall 95\% amino acid sequence identity.

The longest reading frame for mHDAC7 encodes a protein of 938 amino acids with an expected molecular mass of $101 \mathrm{kD}$. Sequence alignment of the HDAC4, mHDAC5, and mHDAC7 reveals that these three proteins share extensive sequence homology (Fig. 2A). Overall, mHDAC7 shares $46 \%$ and $42 \%$ amino acid sequence identity to HDAC4 and HDAC5, respectively (Fig. 2B). The carboxy terminal region, which includes a well-conserved histone deacetylase domain (overall 80\% amino acid sequence identity), and the SMRT interacting domain in both HDAC5 and HDAC7 are mapped to this region. Unlike HDAC4, HDAC5, and HDAC7, HDAC6 contains two histone deacetylase domains of decreased conservation followed by a unique sequence including three copies of CXXC zinc finger motif. A Northern blot probed with mHDAC7 revealed high levels of expression of a $4.2-\mathrm{kb}$ transcript in heart and lung, with low levels in skeletal muscle (Fig. 2C).

Previously, HDAC5 has been shown to possess histone deacetylase activity. To test whether mHDAC7 can also function as a histone deacetylase, HA epitopetagged HDAC5 and HDAC7 were expressed in 293 cells, immunoprecipitated, and assayed for their enzymatic ac- tivity in vitro. The immunoprecipitates were incubated with purified ${ }^{3} \mathrm{H}$-labeled acetate histones. The amount of released ${ }^{3} \mathrm{H}$-labeled acetate was measured by scintillation counting (Fig. 2D). Both mHDAC5 and mHDAC7 exhibit HDAC activity (Fig. 2D, lanes 2,4), which is completely abolished by treatment with $100 \mathrm{~nm}$ of TSA (lanes 3,5). Similarly, using Flag epitope-tagged SMRT and $\mathrm{N}-\mathrm{CoR}$, we also demonstrate that both proteins can immunoprecipitate HDAC activity (Fig. 2E).

\section{mHDAC5 and mHDAC7 repress basal transcription}

Transient transfection experiments were conducted to examine the ability of mHDAC5 and mHDAC7 to repress basal transcription in mammalian cells. Fulllength mHDAC5, mHDAC7, and HDAC1 were fused to the GAL4 DBD 1-147. Repression activity was determined by transiently transfecting increasing amounts of the GAL-fusion vectors along with reporter constructs (Fig. 3A). Fold repression was determined relative to the basal transcription activity of the reporter in the presence of the GAL4 DBD. Both mHDAC5 and mHDAC7 gave $>25$-fold repression activity at each concentration tested and were substantially more active than GAL4 DBD-HDAC1.

To further define repression domains of mHDAC5 and mHDAC7, we made a set of deletion and truncation constructs of mHDAC5 and mHDAC7 fused to GAL4 DBD for repression assays. This led to the identification of two autonomous repression domains in mHDAC5 and three repression domains in $\mathrm{mHDAC} 7$. In addition to the deacetylase domain at the carboxyl terminus of mHDAC7, two additional repression domains corresponding to amino acids 2-254 (R1) and 241-533 (R2) were identified (Fig. 3B). Similarly, HDAC5 contains a single repression domain (R1) corresponding to amino acids 2-378 (Fig. 3C). Whereas the repression activities of the deacetylase domain are expected, the potent activities of these additional regions suggest potential new autonomous repressor functions.

\section{SMRT interacts with mHDAC5 and mHDAC7 in vitro and in vivo}

To map the region in SMRT that interacts with HDAC5, a series of SMRT deletion and truncation constructs were generated and tested in a yeast two-hybrid assay. As described earlier, we observed an association between SMRT RD III and RD IV with the carboxy-terminal sequence of HDAC5. In contrast, GAL4 DBD fusions of repression domains I or II (RD I or RD II) failed to show any HDAC5 association. In summary, the minimal HDAC5-interacting domain was mapped to amino acids 1281-1785 (Fig. 4A). HDAC7 also interacts with SMRT in the RD III/RD IV region (data not shown). In a similar experiment, when a GAL DBD-N-CoR (1016-1875), which spans RD III and RD IV was used, a high reporter activity was observed, suggesting that association between SMRT and mHDAC5/7 is also conserved in $\mathrm{N}$ - 


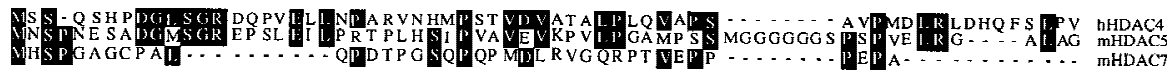

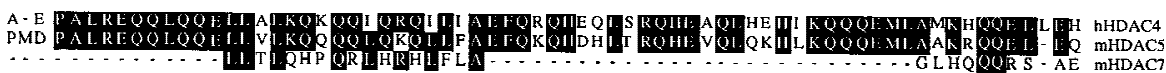

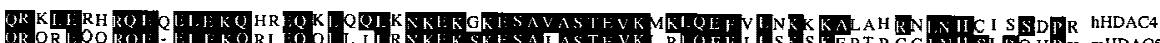
PMRISMDPMP

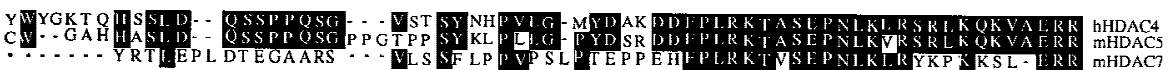

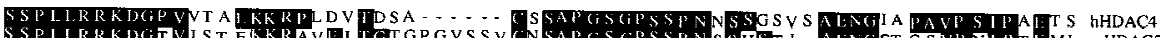

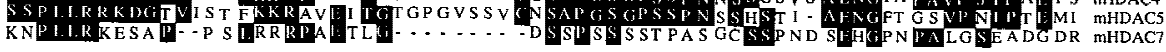

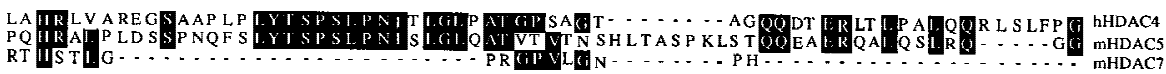

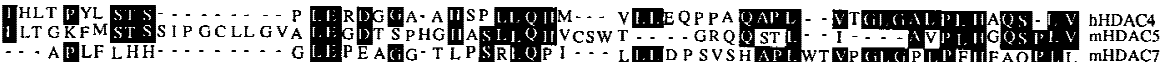

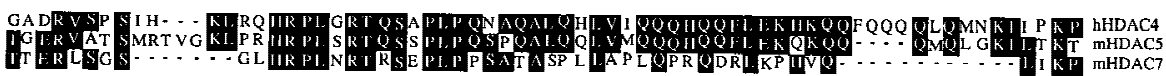

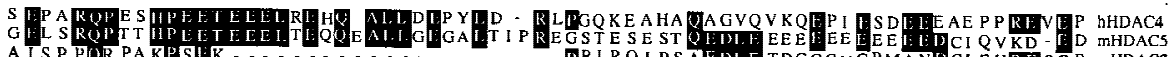

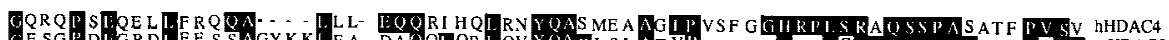

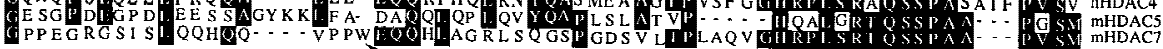

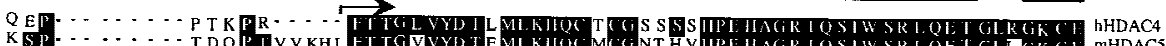

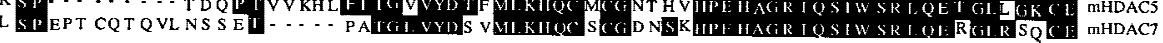

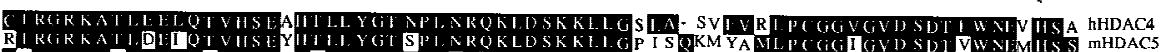

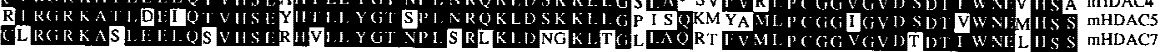

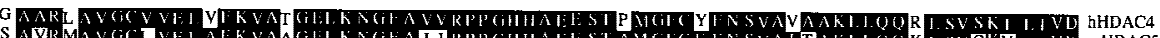
N

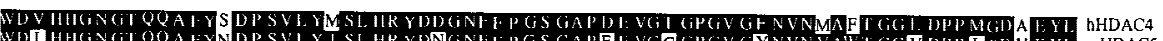

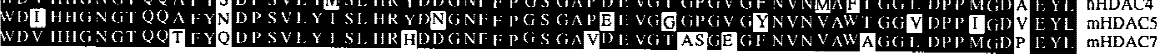

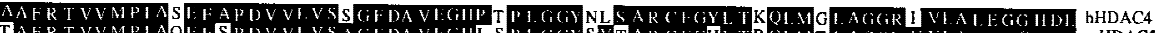

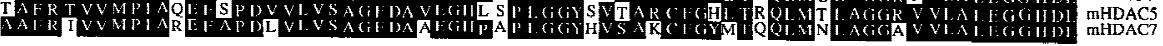

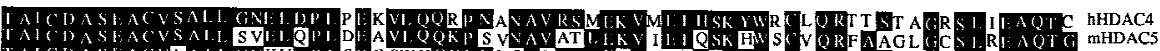

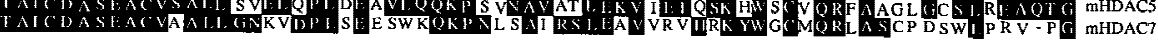

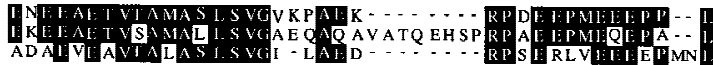

B

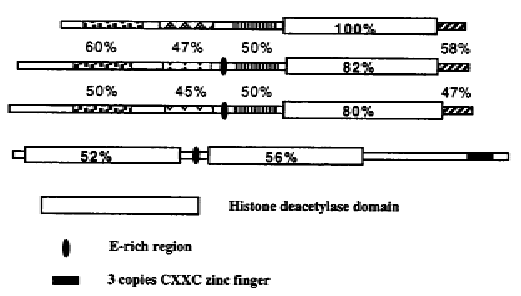

D

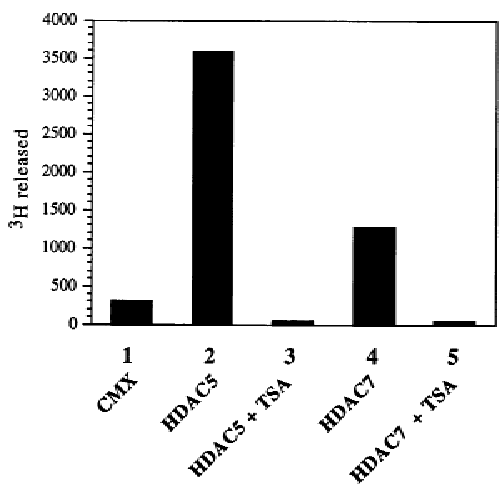

C
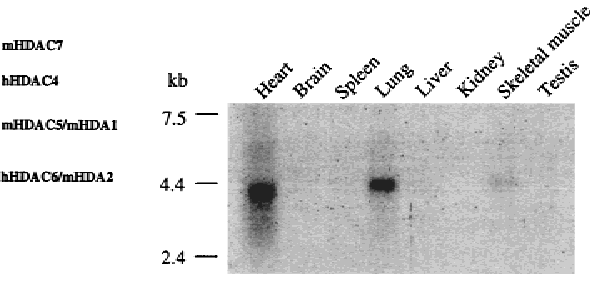

$\mathbf{E}$

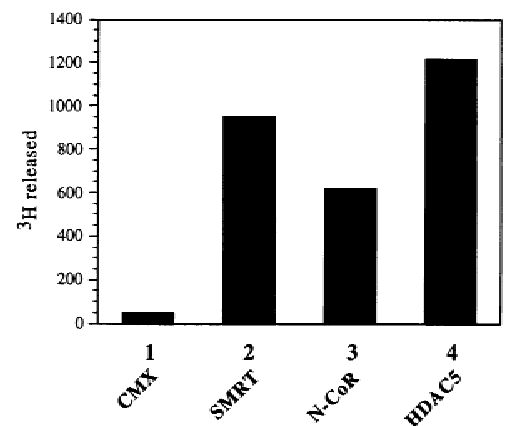

Figure 2. (See facing page for legend.) 
CoR. We also tested whether SMRT sequences other than RD III and RD IV can interact with HDAC1 and mHDAC6 in yeast. Interestingly, no interaction was found (data not shown). A set of deletion and truncation constructs of HDAC5 was also generated to map SMRT interaction domains. Our results indicate that the SMRT-interacting domains reside at the conserved carboxy-terminal region, which includes the deacetylase domain of both HDAC5 and HDAC7 (data not shown).

A GST pull-down assay was used to determine whether mHDAC5 interacts with SMRT in vitro (Fig. 4B). A bacterially expressed GST fusion with the mHDAC5 carboxy-terminal containing histone deacetylase domain was incubated with cell extracts prepared from 293 cells transfected with a SMRT-Flag construct. After extensive washing, retained proteins were separated on an SDS-polyacrylamide gel. Western blot with anti-Flag antibodies was used to detect whether SMRT is present in the pull-down fraction. This data reveals that GST-mHDAC5 can directly bind SMRT or interacts with SMRT-associated proteins.

To confirm the interaction between SMRT and mHDAC5 and mHDAC7 in mammalian cells, coimmunoprecipitation experiments were conducted (Fig. 4C). A total of 293 cells were transfected with plasmids expressing mHDAC5/mHDAC7-HA or both mSMRT-Flag and mHDAC5/mHDAC7-HA. The expression level of mHDACs is equivalent in all samples (data not shown). Cell lysates were prepared and immunoprecipitated with anti-Flag antibodies conjugated to agarose beads. In support of previous results, mHDAC5-HA and mHDAC7HA were precipitated by anti-Flag antibodies only in the presence of SMRT-Flag.

SMRT, mHDAC5, and mHDAC7 colocalize in subnuclear regions

To examine the subcellular localization of the HDAC5 and HDAC7 proteins in CV-1 cells, cells transfected with plasmid expressing YFP-mHDAC5 or mHDAC7 were visualized by fluorescence microscopy and the resulting images were deconvolved with Deltavision2 software. Interestingly, both mHDAC5 (see below) and mHDAC7 were found predominantly in the nucleus with distinct subnuclear dot-like structures (Fig. 5A, left). Furthermore, YFP-mHDAC5 and mHDAC7 staining did not overlap with DAPI staining (Fig. 5A, middle and right). To address the possibility that mHDAC5 and mHDAC7 colocalize, plasmids expressing YFPmHDAC5 and CFP-mHDAC7 were generated and cotransfected into CV-1 cells. We found that mHDAC5 and mHDAC7 colocalized in the same subnuclear compartment (Fig. 5B). We next examined whether mHDAC5 could colocalize with endogenous SMRT. CV-1 cells transfected with YFP-mHDAC5 were subjected to indirect immunofluorescence with anti-SMRT and antiCREB-binding protein (CBP) antibodies, which was used as a control. Whereas mHDAC5 and SMRT were found to completely overlap in subnuclear structures (Fig. 5C, top), mHDAC5 and CBP did not colocalize (Fig. 5C, bottom), strengthening the notion that mHDAC5 and SMRT associate in vivo.

\section{mHDAC7 interacts with $m \operatorname{Sin} 3 A$ in mammalian cells and in yeast}

Given that SMRT interacts with $\mathrm{mSin} 3 \mathrm{~A}$ and that $\mathrm{mSin} 3 \mathrm{~A}$ interacts with $\mathrm{HDAC1}$, we investigated the possibility that mHDAC7 might also interact with mSin3A. To address this issue, we used a coimmunoprecipitation experiment in which 293 cells were transfected with a plasmid expressing mHDAC7-HA. Lysates were prepared from cells with or without mHDAC7-HA expression and incubated with anti-HA antibodi-conjugated agarose beads. Immunoprecipitates were probed with anti-HA, anti-SMRT, and anti-mSin3A antibodies. SMRT and mSin3A were specifically immunoprecipitated in the presence of mHDAC7-HA, indicating that HDAC7, SMRT, and mSin3A can exist as part of a ternary complex (Fig. 6A). To test whether mHDAC7 physically interacts with $\mathrm{mSin} 3 \mathrm{~A}$, we next carried out a yeast two-hybrid assay with a series of GAL DBD-mHDAC7 and $\mathrm{AD}-\mathrm{mSin} 3 \mathrm{~A}$ constructs. We found that $\mathrm{mSin} 3 \mathrm{~A}$ interacts with mHDAC7 through the amino-terminal 192 amino acids containing the first amphipathic helix (PAH1), which apparently differs from the interaction between HDAC1 and mSin3A (Fig. 6B). Furthermore, the $\mathrm{mSin} 3 \mathrm{~A}$ interaction domain was mapped to the carboxyterminal 74 amino acids of HDAC7, which does not include the deacetylase domain (Fig. 6C).

\section{Discussion}

In our attempt to unravel the mechanisms of transcriptional repression by SMRT, we discovered a direct interaction between SMRT/N-CoR and the class II histone

Figure 2. Putative amino acid sequence and tissue distribution of mHDAC7. (A) The deduced amino acid sequence of mHDAC7 and sequence alignment of human HDAC4 and mouse HDAC5 and HDAC7. Sequence alignment of HDAC4, HDAC5, and HDAC7 were performed according to the Jotun Hein method with the DNA STAR program. (Arrow) Beginning of the histone deacetylase domain. (B) Schematic representation of the novel histone deacetylase family. The percentage of homology in each domain is determined by blasting against the amino acid sequence of mHDAC7. (Open rectangle) Histone deacetyalse domain. (Solid ovals) Position of the glutamate-rich (E-rich) regions. (Solid rectangle) Three copies of the zinc finger motif. $(C)$ Expression pattern of mHDAC7 in mouse. Multiple mouse tissue Northern blots were probed with mHDAC7 cDNA. The size marker is indicated. (D) mHDAC5 and mHDAC7 associate with histone deacetylase activity. Whole-cell extracts prepared from cells expressing vector alone, mHDAC5 (lanes 2,3), and mHDAC7 (lanes 4,5) were immunoprecipitated with anti-HA antibodies conjugated agarose beads. Immunoprecipitates were resuspended in deacetylase assay buffer for histone deacetylase assays in the presence (lanes 3,5) or absence (lanes 2,4) of 100 nM trichostatin A. (E) SMRT and N-CoR associate with histone deacetylase activity. 
Kao et al.

Figure 3. HDAC5 and HDAC7 repress basal transcription. (A) HDAC5 and HDAC7 repress basal transcription in transient transfection assays. CV-1 cells were transfected with reporter constructs pCMX- $\beta-$ GAL and pMH100-TK-Luc as well as increasing amounts of plasmids expressing GAL-mHADC5 (lanes 2-4), GAL-mHADC7 (lanes 5-7), and GALHDAC1 (lanes 8-10). (Lane 1) Control. Fold repression activity is shown at top of each bar. $(B)$ mHDAC7 possesses three repression domains. The GAL DBD1-147 was fused to the amino-terminal of a series of truncation constructs of mHDAC7, and $0.1 \mu \mathrm{g}$ of the fusion construct was tested in transient transfection assays for repression activity. Fold repression is shown at top of each bar. Fold repression was determined relative to the basal transcription activity of the reporter in the presence of GAL4 DBD. (C) mHDAC5 contains two repression domains. The assays were carried out the same as described above except that the GAL DBD-mHDAC5 constructs were used.
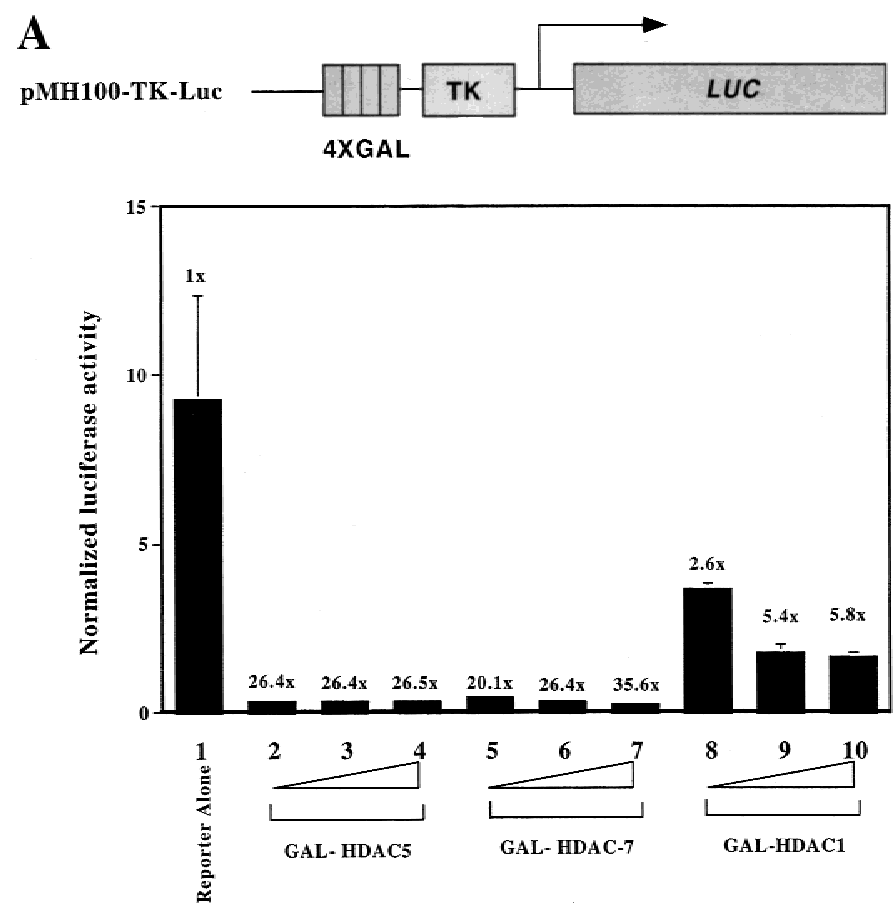

B

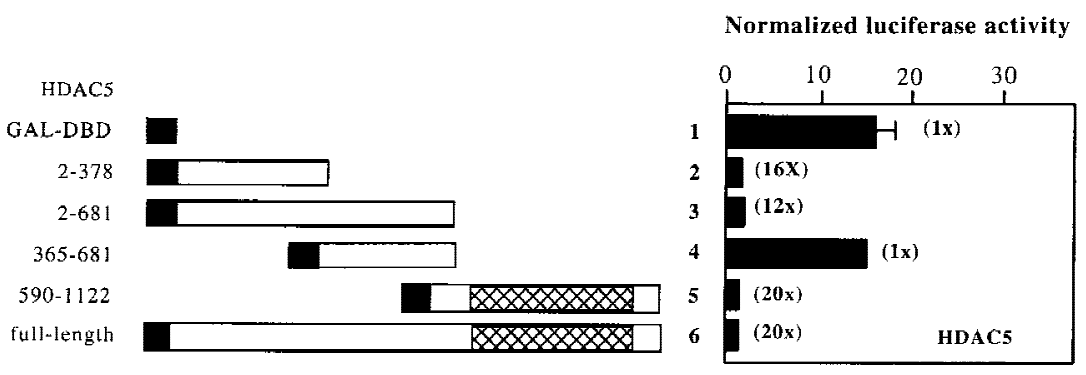

C

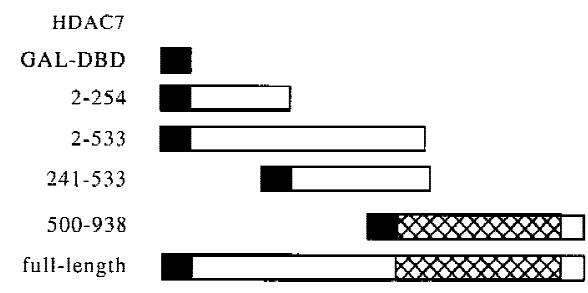

Normalized luciferase activity

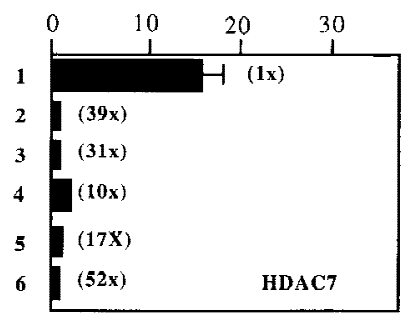

deacetylases HDAC5 and HDAC7. In contrast with class I deacetylases HDAC1 and HDAC3, which do not interact with SMRT/N-CoR, we demonstrate a direct interaction of the corepressors with HDAC5 and HDAC7 through the carboxy-terminal region that includes the deacetylase domain. Furthermore, we demonstrated that HDAC7 interacts with $\mathrm{mSin} 3 \mathrm{~A}$ in mammalian cells as well as in yeast two-hybrid assays. These results provide the first evidence that the silencing mediators SMRT and N-CoR can repress transcription by use of an additional mechanism that involves the recruitment of class II histone deacetylases HDAC5 and HDAC7 (Fig. 7).

\section{The expanding histone deacetylase family}

Seven mammalian histone deacetylases have now been cloned (Verdel et al. 1999; Fischle et al. 1999; Grozinger et al. 1999; this work). These deacetylases can be 
A
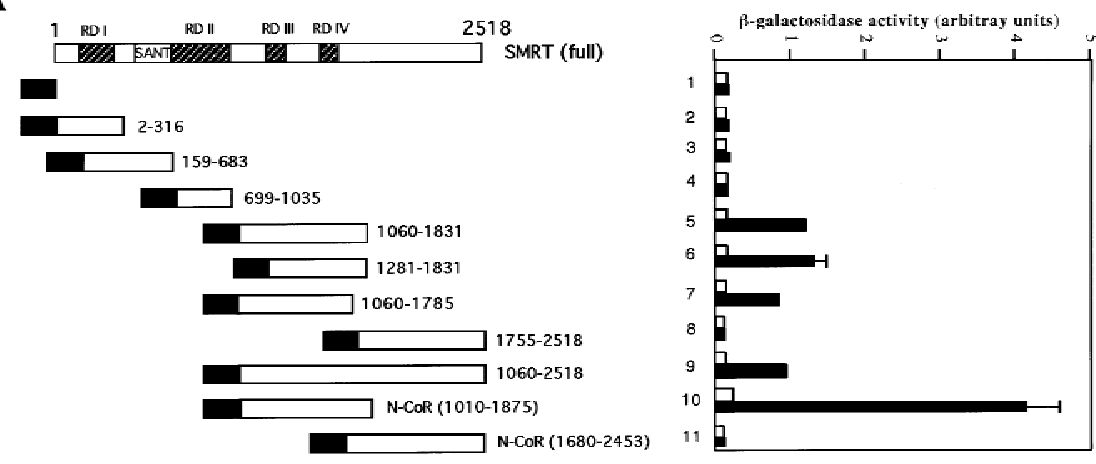

B

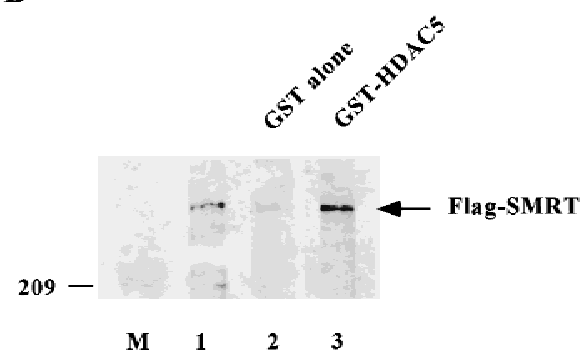

C

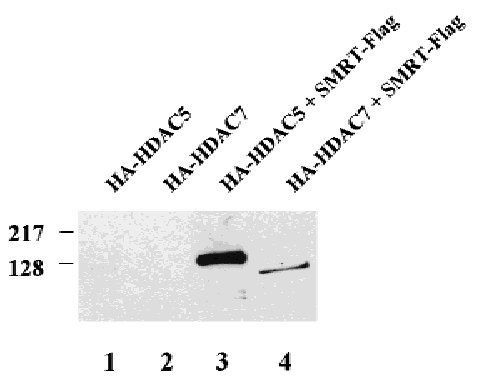

Figure 4. SMRT interacts with mHDAC5 and mHDAC7 in vitro and in vivo. $(A)$ Summary of yeast two-hybrid assays. SMRT contains four repression domains denoted as RD I, RD II, RD III, and RD IV. RD III and RD IV were renamed from SRD I and SRD II, respectively. Note that the HDAC5-interacting domain was mapped to amino acids 1281-1785. Quantitation of the yeast two-hybrid assays are indicated following each construct shown. (Open bars) $\mathrm{AD}$ alone; (solid bars) AD-mHDAC. (B) HDAC5 interacts with SMRT in vitro. (Lane 1) $12.5 \%$ of input, (lane 2) GST alone, (lane 3) GST-HDAC5 (590-1122). (D) SMRT interacts with HDAC5 and HDAC7 in mammalian cells. Anti-Flag antibodies were incubated with whole cell extracts prepared from cells expressing mHDAC5-HA (lane 1), mHDAC7-HA (lane 2), mHDAC5-HA and SMRT-Flag (lane 3), and mHDAC7-HA and SMRTFlag (lane 4). Immunoprecipitates were separated onto a SDS-polyacrylamide gel and Western analysis was carried out with anti-HA antibodies as a probe. The expression level of mHDAC5-HA and mHDAC7-HA is equivalent in all extracts as determined by Western blots (not shown). grouped into two classes on the basis of their structural organization and biochemical characteristics. Class I includes HDAC1, HDAC2, and HDAC3, which appear to be similar to yeast Rpd3 protein. The divergent class II deacetylases include HDAC4, HDAC5, HDAC6, and HDAC7, which resemble the yeast deacetylase Hda1. However, the amino-terminal sequence of HDAC4, HDAC5, and HDAC7 appear to be different from Hda1 and may subserve additional functions. HDAC6 is unique within class II in several ways, including (1) it contains two deacetylase domains; (2) the deacetylase domain is less conserved $(50 \%$ as opposed to $80 \%$ among HDAC4, HDAC5, and HDAC7); (3) HDAC6 does not interact with SMRT (this work). These differences in structure suggest that they may have distinct functional properties. In support of this hypothesis, genetic studies in yeast have demonstrated that a deletion of $R P D 3$, but not $H D A 1$ results in hyperacetylation of the lysine-5 residue of $\mathrm{H} 4$ in several promoters, indicating that $\mathrm{Rpd} 3$ and Hdal may have different substrate specificity (Kadosh and Struhl 1998; Rundlett et al. 1998).

In addition to the potential differences in their biochemical characteristics, class-II HDACs display distinct tissue specificity. For example, HDAC4 is abundant in skeletal muscle and brain (Grozinger et al. 1999), whereas mHDAC5 is enriched in heart /Verdel and Khochbin 1999) and mHDAC6 is expressed highly in testis (Verdel and Khochbin 1999). In this study, we find that mHDAC7 is most abundant in heart and lung and low in skeletal muscle. These observations suggest that these histone deacetylases are not redundant, but rather, have distinct physiological functions.

\section{Structure-function analysis of the novel histone deacetylases}

Using green fluorescence as well as indirect immunofluorescence microscopy, we showed that SMRT, mHDAC5, and mHDAC7 can colocalize to distinct subnuclear structures. This staining pattern differs from other characterized subnuclear structures like PML nuclear bodies as shown in our CBP immunostaining. Although the nature of this subnuclear structure is currently unknown, a similar staining pattern has been reported with HDAC4 (Fischle et al. 1999; Miska et al. 1999). Interestingly, HDAC4 was recently shown to shuttle between the cytoplasm and nucleus in HeLa cells indicating that subcellular localization of HDAC4 is regulated by an active nuclear export process and that nuclear HDAC4 may be required only when cells respond to certain signals (Miska et al. 1999).

We have defined multiple repression domains in mHDAC5 and mHDAC7. The carboxy-terminal region that includes the deacetylase domain of mHDAC5 and mHDAC7 not only interacts with SMRT and mSin3A but also possesses strong repression activity ( 17-fold). This is consistent with the notion that histone deacetylase activity is linked to repression. In addition to the carboxy-terminal deacetylase domain, two repression domains in HDAC7 and one repression domain in HDAC5 were mapped to the amino terminus. This un- 
Kao et al.

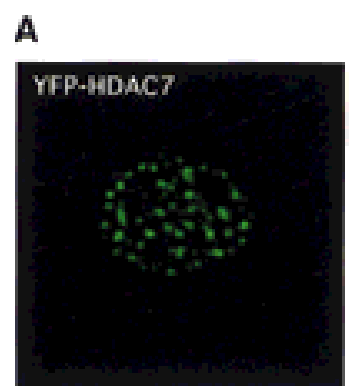

B
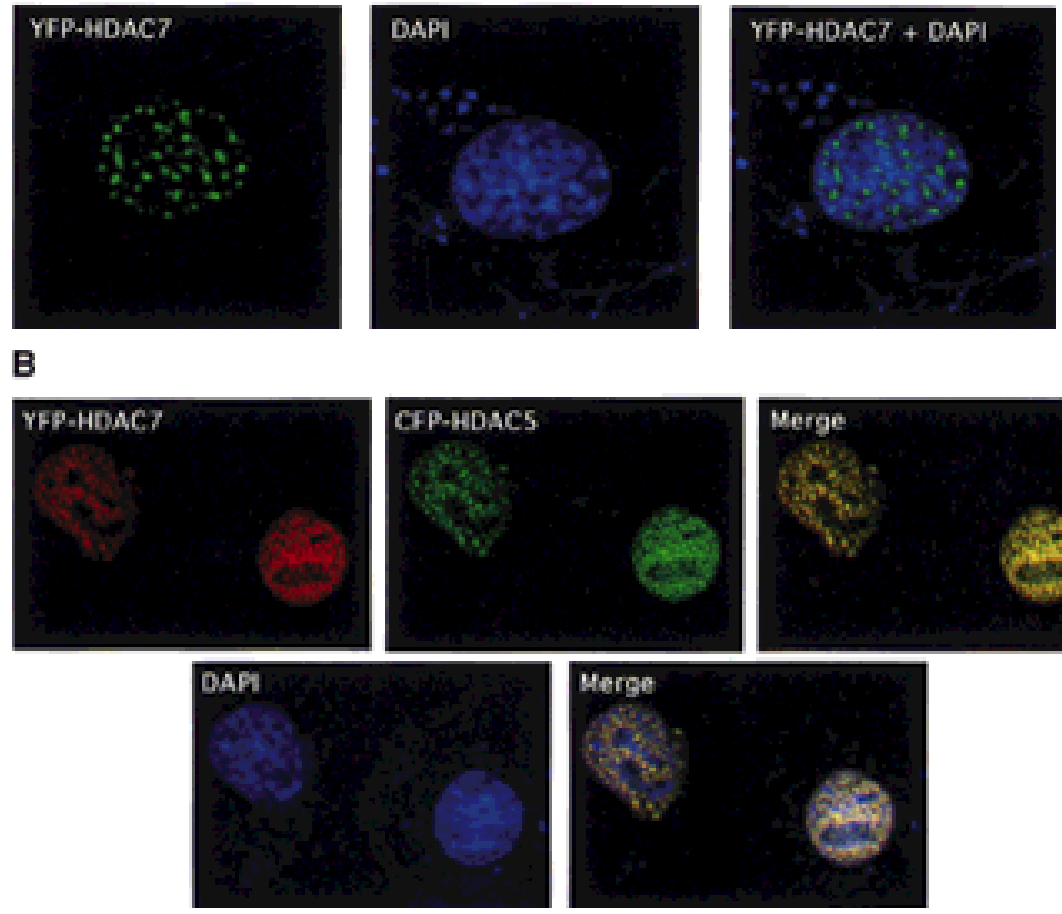

C

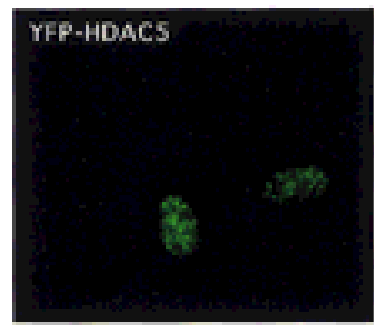

Fure 5. mHDAC5 colocalizes wit dominantly localizes in nucleus with dotlike subnuclear structure. Note that mHDAC7 and DAPI staining are not overlapped. $(B)$ mHDAC5 and mHDAC7 colocalize in the nucleus. $(C)$ mHDAC5 colocalizes with SMRT, but not CBP in CV-1 cells. Note that CBP also gives speckle staining, which represents PML nuclear bodies.

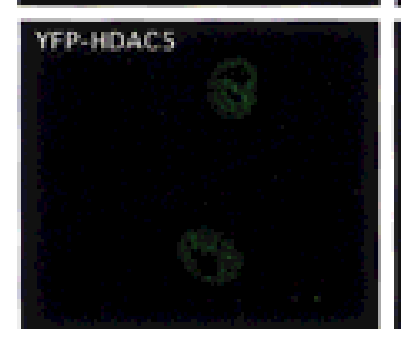

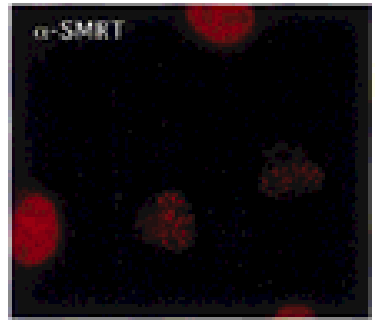

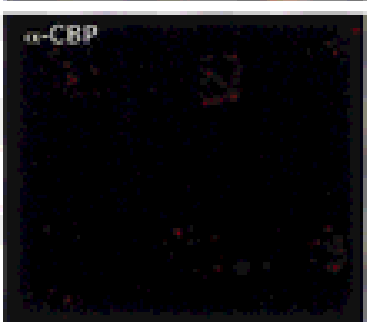

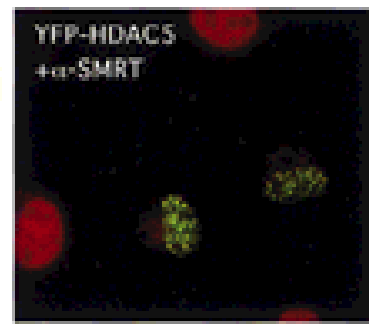
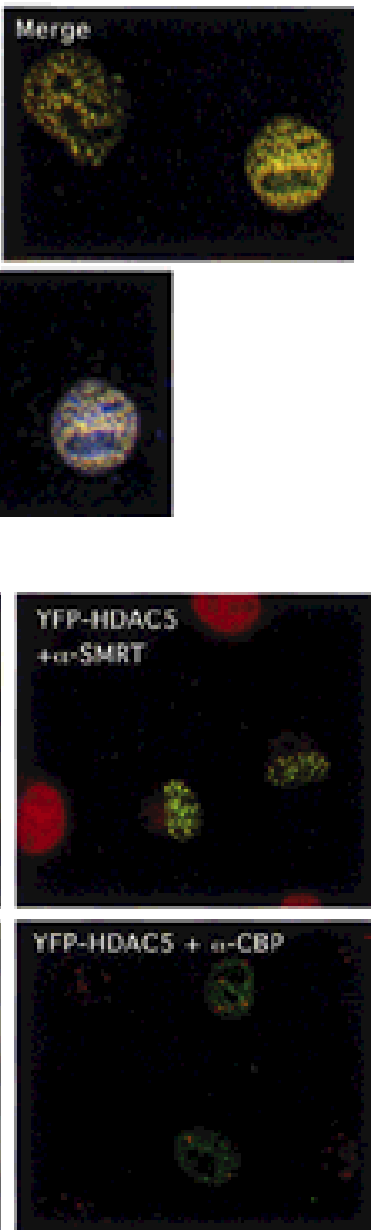

expected observation suggests that deacetylases may repress through additional mechanisms. Alternatively, these domains could recruit other deacetylases to promote the formation of large enzymatic complexes.

\section{The network of protein-protein interactions}

The specificity of the interaction between SMRT and members of the deacetylase family are particularly interesting as the histone deacetylase domains themselves apparently possess distinct features that allow SMRT to recognize one, but not the other. Interestingly, SMRT interactions require an amino acid segment carboxy-terminal to the deacetylase domain of HDAC5 and HDAC7. Those deacetylases that fail to interact with
SMRT (HDAC1, HDAC3, and HDAC6) each lack this segment.

We show here that the HDAC7 interacts with $\mathrm{mSin} 3 \mathrm{~A}$ and that this interaction requires the amino-terminal 192 amino acids of $\mathrm{mSin} 3 \mathrm{~A}$, which is in contrast to previous reports that $\mathrm{HDAC} 1 / \mathrm{HDAC} 2$ associate with PAH3 and PAH4 of mSin3A (Laherty et al. 1997). This interaction was further mapped to the carboxy-terminal region of HDAC7 that does not overlap with the deacetylase domain. The observation that $\mathrm{mSin} 3 \mathrm{~A}$ interacts with HDAC7 is unexpected as class II proteins have not been described to possess this property. Furthermore, in yeast, the histone acetylation pattern of a $\sin 3$ deletion and a hda1 deletion strain are quite different, suggesting that yeast $\operatorname{Sin} 3$ and Hdal function through different ge- 
$\mathbf{A}$

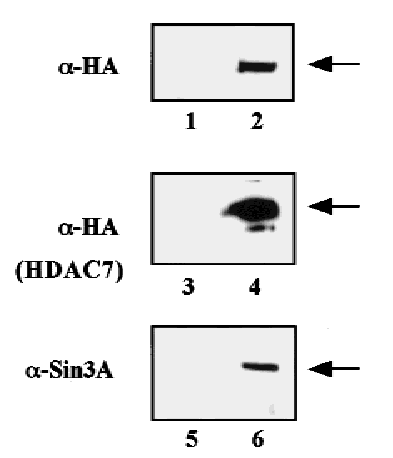

$\alpha$-SMRT

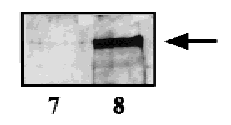

B

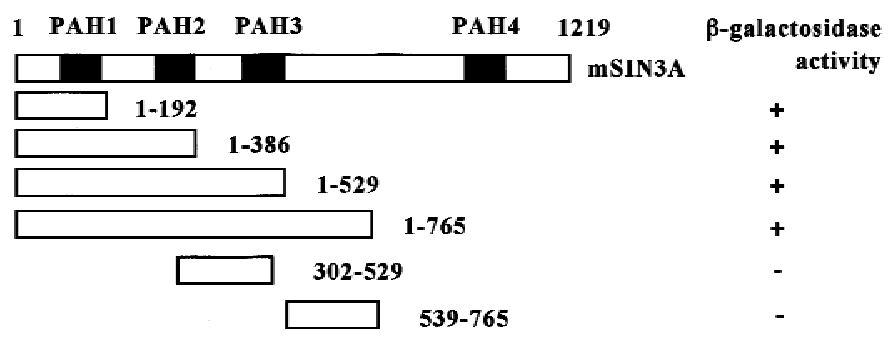

C

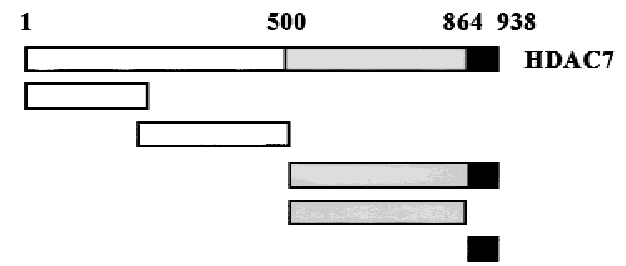

$\beta$-galactosidase activity

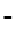

$-$

Figure 6. mHDAC7 complexes with $\mathrm{mSin} 3 \mathrm{~A}$ in yeast and in mammalian cells. (A) mHDAC7 complexes with $\mathrm{mSin} 3 \mathrm{~A}$ in 293 cells. Whole-cell extracts prepared from 293 cells with (lanes 2,4,6,8) or without mHDAC7-HA expression (lanes 1,3,5,7) were incubated with anti-HA antibodies conjugated with agarose beads. Immunoprecipitates were subjected to Western blot analysis and probed with anti-HA antibodies (lanes 3,4), anti-mSin3A antibodies (lanes 5,6), and anti-SMRT antibodies (lanes 7,8). (Lanes 1,2) Whole cell extracts probed with anti-HA antibodies. (B) mHDAC7 interacts with PAH1 of mSin3A in yeast. Plasmids pGBT9-HDAC7 (500-938) and pACTII-mSin3A were cotransformed into yeast Y190 strain and $\beta$-galactosidase lifting assays were performed. $(C)$ The carboxyterminal region (amino acids 864-938) of HDAC7 is required for mSin3A interaction. Plasmids pGBT9-HDAC and pACTII-mSin3A (1-386) were cotransformed into yeast strain Y190 in this experiment.

netic pathways (Rundlett et al. 1998). It is possible that mHDAC7 and/or mSin3A gained the capacity to interact with each other through evolution or that mHDAC4, mHDAC5, and mHDAC7 are more related to other yeast Hda1-related proteins such as Hos1, Hos2, and Hos3 that might genetically or physically interact with Sin3.

\section{Recruitment of multiple repression complexes}

Identification of the complexes containing histone deacetylase activity such as HDAC1/HDAC2, NURD,
mSin3A, MeCP2, and HDAC4 have facilitated our understanding of transcriptional repression (Laherty et al. 1997; Hassig et al. 1997; Nan et al. 1998; Wade et al. 1998; Xue et al. 1998; Grozinger et al. 1999|. Components of these complexes such as mSin3A, SAP30, SAP18, MTA1, and MeCP2 have been shown to repress basal transcription presumably by recruiting histone deacetylase activity, as repression can be abolished by the deacetylase inhibitor Trichostatin A (Hassig et al. 1997; Laherty et al. 1997, 1998; Zhang et al. 1997, 1998; Nan et al. 1998; Xue et al. 1998). RbAp48, which has
A

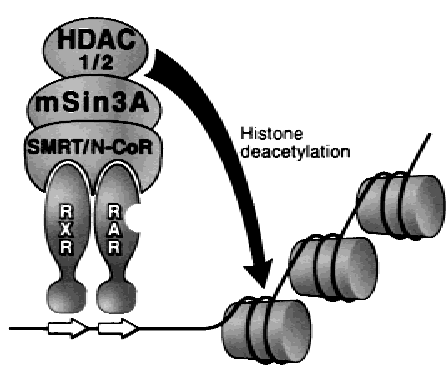

B

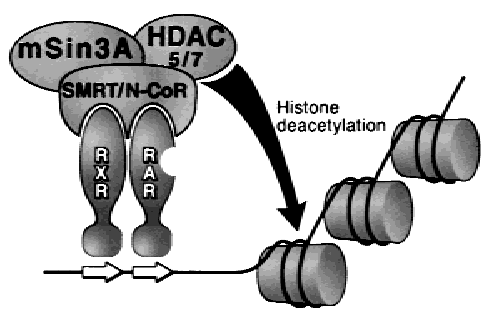

c

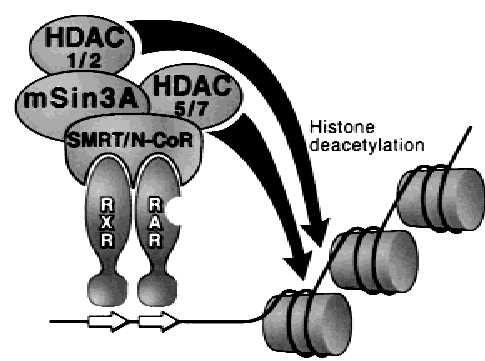

Figure 7. SMRT represses transcription by recruiting both class I and class II histone deacetylase. Repression by a sequence-specific transcription factor such as nuclear receptor heterodimer retinoid X receptor (RXR) and retinoid acid receptor (RAR) is mediated by a SMRT corepressor complex that represses transcription by recruiting both class I and class II histone deacetylases. Models for mechanism of SMRT repression including: (1) SMRT recruits class I deacetylase HDAC1/HDAC2 through direct interaction with mSin3A. (B) SMRT represses transcription by direct interaction with class II deacetylases HDAC5 and HDAC7 (and possibly HDAC4), which also binds to mSin3A through a region different from where HDAC1 binds. (C) SMRT recruits both class I and class II histone deacetylases and $\mathrm{mSin} 3 \mathrm{~A}$. 
been shown to directly contact $\mathrm{H} 4$ and $\mathrm{H} 2 \mathrm{~A}$, is the only factor that has been demonstrated to be a component of histone acetyltransferase, deacetylase, and chromatin assembly complexes. Taken together, these observations directly link histone-modifying enzymes to transcriptional regulation.

Interestingly, $\mathrm{mSin} 3 \mathrm{~A}$ has been shown to associate with corepressors SMRT/N-CoR, HDAC1/HDAC2, MeCP2 complexes, but is not present in the NURD complex. How such restrictions are achieved is not clear but it is suggested that these complexes may substantially differ in function depending on external signals, celltypes, and promoters. In this study, we further demonstrate that corepressors SMRT/N-CoR and $\mathrm{mSin} 3 \mathrm{~A}$ associate with mHDAC7. These multiple protein-protein interactions of repressor complexes are reminiscent of those between coactivators such as $\mathrm{CBP} / \mathrm{p} 300$, PCAF, and the p160 family, suggesting that transcriptional repression, like transcriptional activation, is mediated in part by the local recruitment of a diversity of enzymatic activities to the target promoter.

\section{Materials and methods}

\section{Plasmid construction}

The plasmids pCMX, pCMX-GAL4 DBD, and pMH100-TK-luc have been described (Nagy et al. 1997). Standard PCR amplifications and subcloning techniques were used to generate pCMX carboxy-terminal HA-epitope tagged and GAL4 fusion mHDAC5 and mHDAC7 constructs. All constructs were verified by double-stranded sequencing to confirm identity and reading frame. The vector pCMX-SMRT $\alpha$, which was Flag epitope tagged at the carboxyl terminus, was constructed with cDNAs obtained from a previous screen (Ordentlich et al. 1999), which describes the isolation and characterization of the SMRT $\alpha$ full-length cDNA. Detailed information regarding each construct is available upon request.

\section{Yeast method}

Yeast two-hybrid screen and assays were carried out by the standard lithium acetate method. Mouse embryonic 17-day yeast two-hybrid library (Stratagene) and pGBT9-hSMRT (1060-1831) were cotransformed into yeast strain Y190. Approximately $5 \times 10^{6}$ yeast transformants were screened and selected on yeast minimal medium -Leu-Trp-His plates containing $40 \mathrm{~mm} 3$-AT (Sigma). After 7 days, colonies were picked and confirmed by $\beta$-galactosidase assays. Plasmids were recovered from yeast and retransformed into yeast along with the bait construct. Positive clones were then subjected for sequencing. Liquid $\beta$-galactosidase assays were carried out as described and the number represents an average from duplicate reactions of two colonies.

\section{Full-length cDNA cloning}

Probes used in the isolation of full-length mouse HDAC5 and HDAC7 cDNAs were isolated (EcoRI-BgIII) from the pGAD42.1 vector containing the partial cDNAs obtained from the yeast two-hybrid screen. Accordingly, a mouse brain cDNA $\lambda$ ZAP II library (Stratagene) was screened at low stringency. After two successive rounds, the full-length cDNA sequences for mHDAC5 and mHDAC7 were isolated and sequenced on both strands by standard methods. Accession numbers for mouse HDAC5 and HDAC7 are AF207748 and AF207749, respectively.

\section{Northern blot analysis}

The probe generated with a 1768-bp cDNA EcoRI-HindIII fragment from pCMX-HDAC7 was used to hybridize a mouse poly(A) ${ }^{+}$RNA blot (Clonetech) by standard hybridization and washing protocols.

\section{In vitro interaction assays}

Glutathione $S$-transferase (GST) fusion protein was expressed in Escherichia coli $\mathrm{DH} 5 \alpha$ strain and affinity purified by glutathione-Sepharose 4B beads. In vitro pull-down assays were carried out by incubating GST fusion proteins with nuclear extracts prepared from 293 cells expressing SMRT-Flag protein in G buffer (Kao et al. 1998) for $1 \mathrm{hr}$ at $4^{\circ} \mathrm{C}$ on a nutator. After extensive washes, protein sample buffer was added to retained fractions, boiled, and separated on a 7.5\% SDS-polyacrylamide gel. Western blot analyses probed with anti-HA antibodies were carried out by standard protocols.

\section{Coimmunoprecipitation}

For immunoprecipitations, 293 cells were transfected with 15 $\mu \mathrm{g}$ of the appropriate plasmid with Targefect F1 (Targeting Systems, San Diego, CA). Cells were harvested $48 \mathrm{hr}$ later by lysing in $50 \mathrm{~mm}$ Tris $(\mathrm{pH} 8.0), 150 \mathrm{~mm} \mathrm{NaCl}, 10 \%$ glycerol, $0.5 \%$ Triton X-100, $1 \mathrm{~mm}$ PMSF, and protease inhibitors. Cells were lysed for $15 \mathrm{~min}$ at $4^{\circ} \mathrm{C}$, scraped and centrifuged for $15 \mathrm{~min}$ at $13,000 \mathrm{rpm}$. Supernatant was kept as whole cell extract. After preclearing by incubation with A/G agarose (Santa Cruz), immunoprecipitations were carried out with either HA-agarose (Santa Cruz) or M2-agarose (Sigma) and proceeded for $2 \mathrm{hr}$ at $4^{\circ} \mathrm{C}$. Beads were washed three to four times in lysis buffer without Triton for histone deacetylase assays, and PBS with $0.1 \%$ NP-40 for coimmunoprecipitations. For coimmunoprecipitations, samples were boiled in SDS loading buffer, separated on SDS-polyacrylamide gels, transferred to nitrocellulose membrane, and probed with the appropriate antibodies. The cells were lysed in NET-N buffer in Figure 6.

\section{Histone deacetylase assays}

Histone deacetylase assays were performed according to Heinzel et. al. (1997). Briefly, 60,000 cpm of ${ }^{3} \mathrm{H}$-labeled histones was incubated with immunoprecipitates for $2 \mathrm{hr}$ at $37^{\circ} \mathrm{C}$. Reactions were stopped by addition of acetic acid/HCL to a final concentration of $0.12 / 0.72 \mathrm{~N}$ and extracted with 2 volumes of ethyl acetate. Samples were centrifuged and the supernatant was counted in a scintillation counter. Each reaction represents approximately one-third of a transfected $10 \mathrm{~cm}$ plate of cells. Histone deacetylase activity was carried out in the absence and presence of $100 \mathrm{~nm}$ of TSA.

\section{Green fluorescence and immunofluorescence microscopy}

CV-1 cells were plated into two-well chamber slides (Nunc) and transfected with Targefect F1 (Targeting Systems). After 48 hr, cells were washed in PBS, fixed in $3.7 \%$ paraformaldehyde, and permeabilized with $1 \%$ Triton X-100. For immunostaining, fixed cells were incubated with antibodies against SMRT (Affinity Bioreagents) and CBP (Upstate Biotechnology) for $1 \mathrm{hr}$, washed, and incubated with secondary antibody (Cy3 or Cy5) 
for $1 \mathrm{hr}$. Cells were washed and mounted with Permount. DAPI was included in the final wash to visualize nuclei. Images were visualized with an Olympus 1 X70 inverted system microscope equipped with CCD. The resulting images were deconvolved with Deltavision2 software.

\section{Transient transfection}

Monkey CV-1 cells were grown in DMEM supplemented with $10 \%$ FBS, $50 \mathrm{U} / \mathrm{ml}$ penicillin $\mathrm{G}$, and $50 \mu \mathrm{g} / \mathrm{ml}$ streptomycin sulfate at $37^{\circ} \mathrm{C}$ in $7 \% \mathrm{CO}_{2}$. CV-1 cells $160 \%-70 \%$ confluence, 48-well plate) were cotransfected with 16.6-66.6 ng of pCMXGAL4 and pCMXGAL4-HDAC constructs, $100 \mathrm{ng}$ of pMH100-TK-Luc, and $100 \mathrm{ng}$ of pCMX-LacZ in $200 \mu \mathrm{l}$ of DMEM containing $10 \%$ FBS by the $N$-[1-(2,3-dioleoyloxy)propyl]- $N, N, N$-trimethylammonium methylsulfate (DOTAP)-mediated procedure. The amount of DNA in each transfection was kept constant by addition of pCMX. After $24 \mathrm{hr}$, the medium was replaced and cells were harvested and assayed for luciferase activity $36-48 \mathrm{hr}$ after transfection. The luciferase activity was normalized by the level of $\beta$-galactosidase activity. Each transfection was preformed in triplicate and repeated at least three times.

\section{Acknowledgments}

We thank Dr. Schreiber for the hHDAC3 cDNA and Dr. Khochbin for the mHDA2 cDNA; the sequencing facilities at the Salk Institute; C.-T. Tsai, M. Tini, C.-H. Lee for comments on the manuscript; Lita Ong and Elaine Stevens for administrative assistance. We acknowledge C. Simon, E. Banayo, J. Malchiodi, and H. Juguilon for their technical assistance. H.-Y.K. is a fellow of the Leukemia Society of America. M.D. is a C.J. Martin Research fellow from the National Health and Medical Research Foundation of Australia. P.O. is supported by the George E. Hewitt Foundation for Medical Research. R.M.E. is an Investigator of the Howard Hughes Medical Institute at the Salk Institute for Biological Studies and March of Dimes Chair in Molecular and Developmental Biology. This work was supported by NIH grants HD27183, GM 26444, and program project CA54418.

The publication costs of this article were defrayed in part by payment of page charges. This article must therefore be hereby marked "advertisement" in accordance with 18 USC section 1734 solely to indicate this fact.

\section{References}

Alland, L., R. Muhle, H. Hou, Jr., J. Potes, L. Chin, N. SchreiberAgus, and R.A. DePinho. 1997. Role for N-CoR and histone deacetylase in Sin3-mediated transcriptional repression. $\mathrm{Na}$ ture 387: 49-55.

Asahara, H., S. Dutta, H.-Y. Kao, R.M. Evans, and M. Montminy. 1999. Pbx/Hox heterodimers recruit co-activator/corepressor complexes in an isoform-specific manner. Mol. Cell. Biol. 4: 8219-8225.

Bailey, P., M. Downes, P. Lau, J. Harris, S.L. Chen, Y. Hamamori, V. Sartorelli, and G.E. Muscat. 1999. The nuclear receptor corepressor N-CoR regulates differentiation: N-CoR directly interacts with MyoD. Mol. Endocrinol. 13: 11551168.

Bannister, A.J. and T. Kouzarides. 1996. The CBP co-activator is a histone acetyltransferase. Nature 384: 641-643.

Blanco, J.C., S. Minucci, J. Lu, X.J. Yang, K.K. Walker, H. Chen, R.M. Evans, Y. Nakatani, and K. Ozato. 1998. The histone acetylase PCAF is a nuclear receptor coactivator. Genes \& Dev. 12: 1638-1651.

Carmen, A.A., S.E. Rundlett, and M. Grunstein. 1996. HDA1 and HDA 3 are components of a yeast histone deacetylase (HDA) complex. J. Biol. Chem. 271: 15837-15844.

Chen, H., R.J. Lin, R.L. Schiltz, D. Chakravarti, A. Nash, L. Nagy, M.L. Privalsky, Y. Nakatani, and R.M. Evans. 1997. Nuclear receptor coactivator ACTR is a novel histone acetyltransferase and forms a multimeric activation complex with P/CAF and CBP/p300. Cell 90: 569-580.

Chen, J.D. and R.M. Evans. 1995. A transcriptional co-repressor that interacts with nuclear hormone receptors. Nature 377: 454-457.

Dhordain, P., O. Albagli, R.J. Lin, S. Ansieau, S. Quief, A. Leutz, J.P. Kerckaert, R.M. Evans, and D. Leprince. 1997. Corepressor SMRT binds the BTB/POZ repressing domain of the LAZ3/BCL6 oncoprotein. Proc. Nat1. Acad. Sci. 94: 10762 10767.

Doetzlhofer, A., H. Rotheneder, G. Lagger, M. Koranda, V. Kurtev, G. Brosch, E. Wintersberger, and C. Seiser. 1999. Histone deacetylase 1 can repress transcription by binding to Sp1. Mol. Cell. Biol. 19: 5504-5511.

Dressel, U., D. Thormeyer, B. Altincicek, A. Paululat, M. Eggert, S. Schneider, A.P. Tenbaum, R. Renkawitz, and A. Baniahmad. 1999. Alien, a highly conserved protein with characteristics of a corepressor for members of the nuclear hormone receptor superfamily. Mol. Cell. Biol. 19: 3383-3394.

Emiliani, S., W. Fischle, C. Van Lint, Y. Al-Abed, and E. Verdin. 1998. Characterization of a human RPD3 ortholog, HDAC3. Proc. Natl. Acad. Sci. 95: 2795-2800.

Fischle, W., S. Emiliani, M.J. Hendzel, T. Nagase, N. Nomura, W. Voelter, and E. Verdin. 1999. A new family of human histone deacetylases related to Saccharomyces cerevisiae HDA1p. J. Biol. Chem. 274: 11713-11720.

Grozinger, C.M., C.A. Hassig, and S.L. Schreiber. 1999. Three proteins define a class of human histone deacetylases related to yeast Hdalp. Proc. Natl. Acad. Sci. 96: 4868-4873.

Hassig, C.A., T.C. Fleischer, A.N. Billin, S.L. Schreiber, and D.E. Ayer. 1997. Histone deacetylase activity is required for full transcriptional repression by mSin3A. Cell 89: 341-347.

He, L.Z., F. Guidez, C. Tribioli, D. Peruzzi, M. Ruthardt, A. Zelent, and P.P. Pandolfi. 1998. Distinct interactions of PML-RARalpha and PLZF-RARalpha with co-repressors determine differential responses to RA in APL. Nat. Genet. 18: $126-135$.

Heinzel, T., R.M. Lavinsky, T.M. Mullen, M. Soderstrom, C.D. Laherty, J. Torchia, W.M. Yang, G. Brard, S.D. Ngo, J.R. Davie et al. 1997. A complex containing N-CoR, mSin3 and histone deacetylase mediates transcriptional repression. $\mathrm{Na}$ ture 387: 43-48.

Hong, H., K. Kohli, A. Trivedi, D.L. Johnson, and M.R. Stallcup. 1996. GRIP1, a novel mouse protein that serves as a transcriptional coactivator in yeast for the hormone binding domains of steroid receptors. Proc. Natl. Acad. Sci. 93: 49484952.

Hong, S.H., G. David, C.W. Wong, A. Dejean, and M.L. Privalsky. 1997. SMRT corepressor interacts with PLZF and with the PML-retinoic acid receptor alpha (RARalpha) and PLZFRARalpha oncoproteins associated with acute promyelocytic leukemia. Proc. Natl. Acad. Sci. 94: 9028-9033.

Horlein, A.J., A.M. Naar, T. Heinzel, J. Torchia, B. Gloss, R. Kurokawa, A. Ryan, Y. Kamei, M. Soderstrom, C.K. Glass et al. 1995. Ligand-independent repression by the thyroid hormone receptor mediated by a nuclear receptor co-repressor. Nature 377: 397-404.

Huynh, K.D. and V.J. Bardwell. 1998. The BCL-6 POZ domain 
and other POZ domains interact with the co- repressors $\mathrm{N}$ CoR and SMRT. Oncogene 17: 2473-2484.

Iavarone, A. and J. Massague. 1999. E2F and histone deacetylase mediate transforming growth factor beta repression of cdc25A during keratinocyte cell cycle arrest. Mol. Cell. Biol. 19: 916-922.

Kadosh, D. and K. Struhl. 1998. Targeted recruitment of the Sin3-Rpd3 histone deacetylase complex generates a highly localized domain of repressed chromatin in vivo. Mol. Cell. Biol. 18: 5121-5127.

Kamei, K., L. Xu, T. Heinzel, J. Torchia, R. Kurokawa, B. Gloss, S.-C. Lin, R.A. Heyman, D.W. Rose, C.K. Glass, and M.G. Rosenfeld. 1996. A CBP Integrator complex mediates transcriptional activation and AP-1 inhibition by nuclear receptors. Cell 85: 403-414.

Kao, H.-Y., P. Ordentlich, N. Koyano-Nakagawa, Z. Tang, M. Downes, C.R. Kintner, R.M. Evans, and T. Kadesch. 1998. A histone deacetylase corepressor complex regulates the Notch signal transduction pathway. Genes \& Dev. 12: 22692277.

Laherty, C.D., W.M. Yang, J.M. Sun, J.R. Davie, E. Seto, and R.N. Eisenman. 1997. Histone deacetylase associated with the mSin 3 corepressor mediate Mad transcriptional repression. Cell 89: 349-356.

Laherty, C.D., A.N. Billin, R.M. Lavinsky, G.S. Yochum, A.C. Bush, J.M. Sun, T.M. Mullen, J.R. Davie, D.W. Rose, C.K. Glass et al. 1998. SAP30, a component of the $\mathrm{mSin} 3$ corepressor complex involved in N-CoR-mediated repression by specific transcription factors. Mol. Cell 2: 33-42.

Lin, R.J., L. Nagy, S. Inoue, W. Shao, W.H. Miller, Jr., and R.M. Evans. 1998. Role of the histone deacetylase complex in acute promyelocytic leukaemia. Nature 391: 811-814.

Mangelsdorf, D.J. and R.M. Evans. 1995. The RXR heterodimers and orphan receptors. Cell 83: 841-850.

Mangelsdorf, D.J., C. Thummel, M. Beato, P. Herrlich, G. Schutz, K. Umesono, B. Blumberg, P. Kastner, M. Mark, P. Chambon, and R.M. Evans. 1995. The nuclear receptor superfamily: The second decade. Cell 83: 835-839.

Miska, E.A., C. Karlsson, E. Langley, S.J. Nielsen, J. Pines, and T. Kouzarides. 1999. HDAC4 deacetylase associates with and represses the MEF2 transcription factor. EMBO J. 18: 5099-5107.

Muto, A., H. Hoshino, L. Madisen, N. Yanai, M. Obinata, H. Karasuyama, N. Hayashi, H. Nakauchi, M. Yamamoto, M. Groudine, and K. Igarashi. 1998. Identification of Bach2 as a B-cell-specific partner for small maf proteins that negatively regulate the immunoglobulin heavy chain gene 3 ' enhancer. EMBO J. 17: 5734-5743.

Nagy, L., H.-Y. Kao, D. Chakravarti, R.J. Lin, C.A. Hassig, D.E. Ayer, S.L. Schreiber, and R.M. Evans. 1997. Nuclear receptor repression mediated by a complex containing SMRT, mSin3A, and histone deacetylase. Cell 89: 373-380.

Nan, X., H.H. Ng, C.A. Johnson, C.D. Laherty, B.M. Turner, R.N. Eisenman, and A. Bird. 1998. Transcriptional repression by the methyl-CpG-binding protein MeCP2 involves a histone deacetylase complex. Nature 393: 386-389.

Ogryzko, V.V., R.L. Schiltz, V. Russanova, B.H. Howard, and Y. Nakatani. 1996. The transcriptional coactivators p300 and CBP are histone acetyltransferases. Cell 87: 953-959.

Onate, S.A., S.Y. Tsai, M.J. Tsai, and B.W. O'Malley. 1995. Sequence and characterization of a coactivator for the steroid hormone receptor superfamily. Science 270: 1354-1357.

Ordentlich, P., M. Downes, W. Xie, A. Genin, N.B. Spinner, and R.M. Evans. 1999. Unique forms of human and mouse nuclear receptor corepressor SMRT. Proc. Natl. Acad. Sci. 96: $2639-2644$.
Radkov, S.A., R. Touitou, A. Brehm, M. Rowe, M. West, T. Kouzarides, and M.J. Allday. 1999. Epstein-Barr virus nuclear antigen 3C interacts with histone deacetylase to repress transcription. J. Virol. 73: 5688-5697.

Rundlett, S.E., A.A. Carmen, R. Kobayashi, S. Bavykin, B.M. Turner, and M. Grunstein. 1996. HDA1 and RPD3 are members of distinct yeast histone deacetylase complexes that regulate silencing and transcription. Proc. Natl. Acad. Sci. 93: $14503-14508$.

Rundlett, S.E., A.A. Carmen, N. Suka, B.M. Turner, and M. Grunstein. 1998. Transcriptional repression by UME6 involves deacetylation of lysine 5 of histone $\mathrm{H} 4$ by RPD3. $\mathrm{Na}$ ture 392: 831-835.

Spencer, T.E., G. Jenster, M.M. Burcin, C.D. Allis, J. Zhou, C.A. Mizzen, N.J. McKenna, S.A. Onate, S.Y. Tsai, M.J. Tsai, and B.W. O'Malley. 1997. Steroid receptor coactivator-1 is a histone acetyltransferase. Nature 389: 194-198.

Torchia, J., D.W. Rose, J. Inostroza, Y. Kamei, S. Westin, C.K. Glass, and M.G. Rosenfeld. 1997. The transcriptional coactivator $\mathrm{p} / \mathrm{CIP}$ binds $\mathrm{CBP}$ and mediates nuclear-receptor function. Nature 387: 677-684.

Verdel, A. and S. Khochbin. 1999. Identification of a new family of higher eukaryotic histone deacetylases. Coordinate expression of differentiation-dependent chromatin modifiers. J. Biol. Chem. 274: 2440-2445.

Wade, P.A., P.L. Jones, D. Vermaak, G.J. Veenstra, A. Imhof, T. Sera, C. Tse, H. Ge, Y.B. Shi, J.C. Hansen, and A.P. Wolffe. 1998. Histone deacetylase directs the dominant silencing of transcription in chromatin: Association with MeCP2 and the Mi-2 chromodomain SWI/SNF ATPase. Cold Spring Harb. Symp. Quant Biol. 63: 435-445.

Wong, C.W. and M.L. Privalsky. 1998. Components of the SMRT corepressor complex exhibit distinctive interactions with the POZ domain oncoproteins PLZF, PLZF-RARalpha, and BCL-6. J. Biol. Chem. 273: 27695-27702.

Xue, Y., J. Wong, G.T. Moreno, M.K. Young, J. Cote, and W. Wang. 1998. NURD, a novel complex with both ATP-dependent chromatin-remodeling and histone deacetylase activities. Mol. Cell 2: 851-861.

Yang, W.M., C. Inouye, Y. Zeng, D. Bearss, and E. Seto. 1996. Transcriptional repression by YY1 is mediated by interaction with a mammalian homolog of the yeast global regulator RPD3. Proc. Nat1. Acad. Sci. 93: 12845-12850.

Yang, X.J., V.V. Ogryzko, J. Nishikawa, B.H. Howard, and Y. Nakatani. 1996. A p300/CBP-associated factor that competes with the adenoviral oncoprotein E1A. Nature 382: 319-324.

Yao, T.-P., G. Ku, N. Zhou, R. Scully, and D.M. Livingston. 1996. The nuclear hormone receptor coactivator SRC-1 is a specific target of p300. Proc. Natl. Acad. Sci. 93: 10626-10631.

Zamir, I., H.P. Harding, G.B. Atkins, A. Horlein, C.K. Glass, M.G. Rosenfeld, and M.A. Lazar. 1996. A nuclear hormone receptor corepressor mediates transcriptional silencing by receptors with distinct repression domains. Mol. Cell. Biol. 16: 5458-5465.

Zhang, Y., R. Iratni, H. Erdjument-Bromage, P. Tempst, and D. Reinberg. 1997. Histone deacetylases and SAP18, a novel polypeptide, are components of a human Sin 3 complex. Cell 89: 357-364.

Zhang, Y., Z.W. Sun, R. Iratni, H. Erdjument-Bromage, P. Tempst, M. Hampsey, and D. Reinberg. 1998. SAP30, a novel protein conserved between human and yeast, is a component of a histone deacetylase complex. Mol. Cell 1: 10211031. 


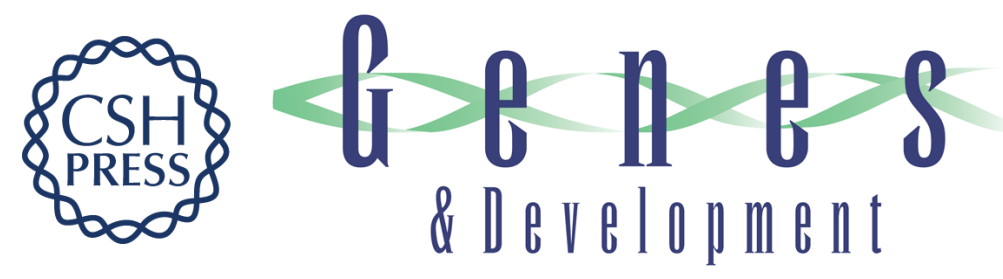

\section{Isolation of a novel histone deacetylase reveals that class I and class II deacetylases promote SMRT-mediated repression}

Hung-Ying Kao, Michael Downes, Peter Ordentlich, et al.

Genes Dev. 2000, 14:

Access the most recent version at doi:10.1101/gad.14.1.55

References

This article cites 52 articles, 24 of which can be accessed free at: http://genesdev.cshlp.org/content/14/1/55.full.html\#ref-list-1

License

Email Alerting

Receive free email alerts when new articles cite this article - sign up in the box at the top Service right corner of the article or click here.

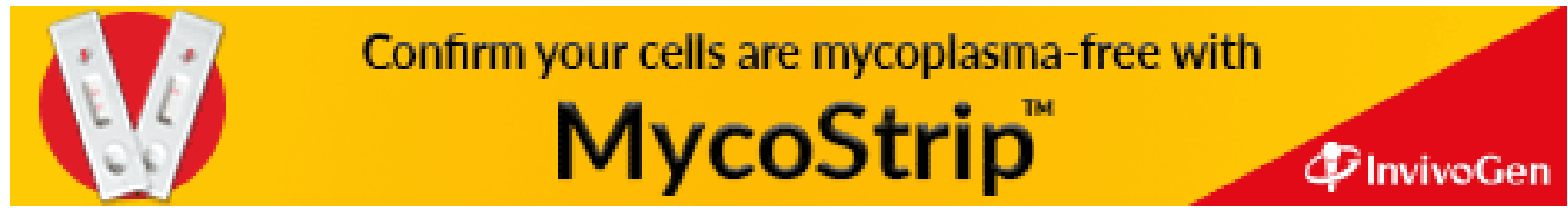

